\title{
Design of an Agent-Based Traffic Control System
}

\author{
Adegoke Ojeniyi, Taye Aro, Ang Moon Thiak
}

\begin{abstract}
Traffic control system is an imperative instrument in traffic management and smart urban development. However, most of the current traffic control systems cannot intercommunicate nor interact with each other. Most importantly, none of these systems are proactive and reactive to their immediate traffic environment. Thus, this study explores the design of an agent-based traffic control system where traffic lights can interact and inter-communicate to take prompt traffic control decisions within a traffic area. The study presents an agent-based traffic control system known as ATC. ATC system design was done using Design Science Research Process Model while the system was evaluated using qualitative research methodology. The study argues that there is need for traffic control system to be more reactive and proactive to their immediate traffic environment in order to limit traffic jam in urban areas.
\end{abstract}

Index Terms: Agent-based system, agent-based traffic system, interactive system, traffic control, urban traffic control.

\section{INTRODUCTION}

The recent positive increases in urbanization, improved transport infrastructure in Abeokuta city and traffic jam generate an urgent need to operate the available system of transportation with maximum efficiency. Hence, the mismanagement of traffic has been identified as one of the major causes of time wastage on our road today. Traffic control can only be seen as effective when it has the ability to react to changes in traffic patterns, however, recent days traffic light control systems in Nigeria and most developing countries are timed and run according to those times only. As a result, has caused hundreds of noticeable problems among which are an increased safety risk, negative impact on the populace's mental wellbeing, environmental pollution and frequent road accident. These problems can only be solved when the traffic control system can communicate with each other. This will not make the traffic control system to be a stand-alone system but an intelligent interactive and reactive system. Intelligent signal control systems must have the capability to optimize the traffic flow by adjusting the traffic lights and coordinate operation between each signal in order to increase the person and vehicular throughput and

\footnotetext{
Revised Manuscript Received on September 22, 2019.

Adegoke Ojeniyi, Head of ArtifiDepartment of Computer Science, KolaDaisi University, Ibadan, Nigeria.

Taye Aro, Department of Computer Science, KolaDaisi University, Ibadan, Nigeria.

Ang Moon Thiak, Othman Yeop Abdullah (OYA) Graduate School of Business, Universiti Utara Malaysia.
}

minimize the delay. As a result, a fully pro-active agent-based system is in no doubt a very useful option for intelligent urban traffic control. Therefore, this paper investigates factors responsible

for traffic congestion in the Abeokuta City and presented an Agent-based traffic control design which will reduce traffic congestions in Abeokuta City.

\section{RELATED WORK}

As Traffic lights to ensure that traffic and pedestrians move as smoothly, and safely as possible requires more than light control and coordination [1]. A variety of different control systems are used to accomplish this, ranging from simple clockwork mechanisms to sophisticated computerized control and coordination systems that self - adjust to minimize delay for people using the road. Traffic control devices are markers, signs and signal devices used to inform, guide and control traffic, including pedestrians, motor vehicle drivers, and bicyclists. These devices are usually placed adjacent, over or along the highways, roads, traffic facilities and other public areas that require traffic control. Thus, traffic control is usually in-use in urban areas which gave birthed to the idea of Urban Traffic Control (UTC) system [1].

UTC systems specially implemented to control, manage, coordinate and segregate the flow of traffic on a road network with the aid of signaling display [2]. A plan is to strategize for the coordination and segregation of traffic based on historical traffic data and the current traffic signal time interval within the road network while introducing a system to link the derived signal measurement electronically [3]. A traffic-responsive signal control system consists of two basic ideas, the objective function which is generally to reduce the travel time and stops, and the internal/external constraints which may include the current and previous traffic signal adjusting settings. Based on control strategies, UTC systems can be classified into seven which include the following:

\section{A.Fixed time Systems}

This method has gained wide recognition and implementations amongst several UTC projects and development schemes. However, the waiting time in this type of system is solely determined by the network designer, the different arterial road is subjected to different delay time objectives based on the perception of the designer [4]. This timing due to the scope of management may be biased against the main traffic movements, traffic can be minimized by adjusting the splits at critical

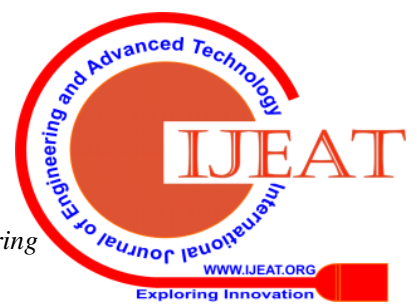


junctions. Also, the cycle time can be kept low to reduce total capacity and extra stages can be introduced at junctions to reduce the green time available for the main traffic movements.

Fixed time systems typically use pre-calculated timing plans, as a result, cannot respond dynamically to alternating demand of road traffic network, they also cannot respond automatically to incidents, such as accidents, which will reduce the number of vehicular movement per time. In addition, they will not respond to changed demand when a route guidance system is working [5]. Fixed time plans are good at implementing fixed strategies when there is a need to limit the frequency of throughput at certain periods of the day.

\section{B.Plan Selection Systems}

This plan has not been proven to be better than the fixed time systems even though they are capable of selecting and changing their current plan strategically based on the current information given from the networks rather than change based on the time of the day [6]. In some important situations, they can be driven to run in a specific manner. However, a wrong plan may be chosen which can alter the benefit of manually choosing a plan against the logic of the automated system, as a result, may cause extra delay to the marginal traffic flow.

\section{C.Plan Generation Systems}

This type of system generates traffic management plan from detector devices which help gather information or data (traffic density impulse from sensing its environment). This system is self-adaptive as it re-adjusts its traffic management operation without the help of the network designer. This system can also react to accident occurrences but may not be allowed to make large enough changes as not to adversely affect its effective performance [7].

\section{D.Local Adaptation}

This system may be viewed as combining the features of both the plan selection and generation systems. Plan selection in a way that an appropriate fixed time plan is run; however, the system is not limited to that plan and have the privilege to terminate or omit some plans for some particular location in the network based on their local demand at a particular time and for the current cycle [8]. Plan generation is its ability to adapt by increasing and reducing the green time interval in some of the arterial network in order to enhance smooth progression in that locality.

\section{E. Traffic Responsive Centralized Systems}

This system, because of its centralized nature, gives out instruction to individual traffic controllers through a central computer. A responsive centralized system makes use of traffic density data inputs from sensor devices [9]. The alternating changes in the measure of flow are transmitted and stored directly into the computer system which in its part takes a decision on the green time interval to be adopted in the various controllers in order to respond to the day-to-day variation in traffic demand, accident occurrences and trends over time [10].

\section{F. Traffic Responsive Systems with Distributed Processing}

This has a sharp semblance to the features of the centralized responsive systems. The major difference is its method of communicating traffic data and information, likewise its control management [11]. In this, any two neighboring controllers can sense their environment and as well communicate by routing their message through wired or wireless interconnections [12], [13]. It works with a route guidance system (which could be a graph centrality calculation embedded in the overall system design) to ensure each controller has the location knowledge of its neighboring and affected controller. This interaction technique has made the system design a little complex.

Although, these studies depict traffic control strategies [14] however, no studies have specifically implemented Agent-based system application on traffic control which is the major contribution of this current study. Hence, this study explores the design of an Agent-based Traffic control system. Agents are hardware or software systems that carry out tasks in complex, continuously changing environments by making decisions without the help of another agent. Agent concept [15], [16] can be viewed as saying "a friend in need is a friend indeed" is the governing body of interactions among the several agents, in multi-agent system, agents work together to analyses an agent's perception of its environment, knowing fully the intended goal of the agent, solving the problem by adjusting their current status in order to help achieve the goal of the needing agent or in a rare case prevent it [17], [18].

\section{METHODOLOGY}

The methodology used in this study is a mixture of Design Science Research Process Model and Qualitative methodology. These two methodologies were used to ensure rich and scholarly results. The Design Science Research Process Model employed in this research work is captured in Fig. 1. 


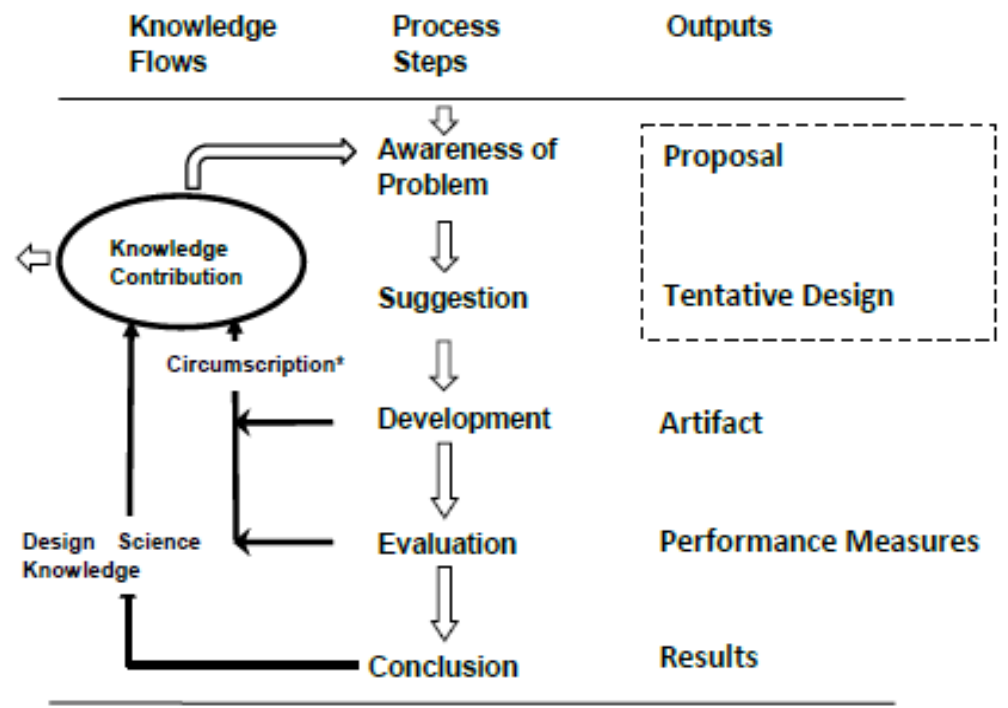

Fig. 1. Design Science Research Process Model (DSR)

The uniqueness of this methodology to other science research methodologies is its contribution of new knowledge in the form of an artifact or system design [19]. The first step in this methodology is the awareness phase whereas traffic problems are the output of this phase. Next is the Suggestion phase which is closely connected with the development phase. Both the Suggestion and Development phases are based on the awareness of traffic problems within Abeokuta City. The Suggestion phase is an important creative stage wherein new functionality is conceived based on a new configuration of either existing or new and existing elements. Whereas the development phase is also known as the implementation stage and technical implementation are carried out at this stage. This is followed by the evaluation phase which made use of qualitative research methodology. The qualitative is based on successive in-depth interviews conducted on six respondents from the traffic regulation agency and compliance (TRACE),

and the Nigerian Police and Road users. Results gotten from this phase is used as information gained in the development and implementation of the system (artifact) are brought together and fed back to another round of Suggestion.

Furthermore, the study area covers from Okemosan, Governor's office (Sub-regions: 60 59'36”N, 30 28' 30”E, Min. elevation: $-3 \mathrm{~m}$, max. elevation: 292m, Land/Water: Mainland) to Kuto (Sub-regions: 60 59'36”N, 30 28' 30'E, Min. elevation: -3m, max. elevation: 292m, Land/Water: Mainland), Abeokuta. Kuto is $2.4 \mathrm{~km}$ away from Okemosan where all these locations are in Southwest Nigeria

\section{FINDINGS AND DISCUSSION}

This section is divided into two namely the Agent-based Traffic Control (ATC) System design and the system evaluation. These two are explained in the following sub-sections

\section{A. ATC System Design}

One of the biggest hurdles in urban societies is to find a lasting solution to the vehicular traffic problem. The design aim and objective as stated earlier was the introduction of traffic lights that will behave like agents by communicating with each other. Sensor devices embedded at the four major intersections in the identified road link will help detect road traffic density and track changes after the agent processing aimed at reducing and regulating vehicular road users. It is also used to trap the results of traffic lights processing outcomes. Each agent has a dedicated sensor interconnected to it by the aid of wired connections. Traffic Lights (Agent): The uniqueness of this study is the view of traffic lights' adaptiveness and intelligent response to environmental road traffic density. The word agent in a traffic light is their ability to work hand-in-hand to execute a particular goal which is the total eradication of traffic congestion/bottleneck in the fast developing metropolis. However, every traffic light used in this system is tagged "AGENT" as a name and these Agents are connected with box controller which embeds control ICs.

For improvement and accurate placement of sensor devices, historical traffic density data sourced from road traffic regulating officials are used for the design of the study. Different and easily accessible indicators which indicate the positioning of the four Agents, the traffic sensor devices are used on the graph generated. All these have helped to obtain the near-optimal design for the project. However, this study makes use of sensors that are to be buried into the pavement especially at every cross junction of the roadway because they are easier to implement, cost-effective and can span for longer period because they are not affected by wear and tear as opposed to those that are installed in vehicles (e.g., GPS) and those connected above or alongside the roadway. Capacitors, transistors including resistors for both powering and enabling the processing of traffic light and its communicating ability learn and report back to the modeler, as usual, a signal in computer simulated language which is presented in Table I. 
Table- I: Summary of Selected Sensors

\begin{tabular}{|c|c|c|}
\hline $\begin{array}{l}\text { Sensors as tracking } \\
\text { equipment in vehicles }\end{array}$ & $\begin{array}{lr}\text { Global Navigation } \\
\text { Satellite System (GNSS) }\end{array}$ & $\begin{array}{l}\text { GPS and Galileo have been among the well-known types help detect the } \\
\text { position and current velocity of automobiles. }\end{array}$ \\
\hline \multirow{5}{*}{$\begin{array}{l}\text { Autonomous } \\
\text { sensors }\end{array}$} & \multirow[b]{2}{*}{$\begin{array}{l}\text { In-roadway } \\
\text { Sensors }\end{array}$} & $\begin{array}{l}\text { Vehicles are been detected by sensing conductive metal in vehicles by } \\
\text { imbibing currents into the object which minimize the loop inductance. }\end{array}$ \\
\hline & & $\begin{array}{l}\text { Magnetic sensors: In conjunction with the inductive-loop detectors, The } \\
\text { agitation of the magnetic field caused by vehicular movement enhances } \\
\text { the sensibility of the sensor. }\end{array}$ \\
\hline & \multirow{3}{*}{$\begin{array}{l}\text { Over-roadway } \\
\text { Sensors }\end{array}$} & $\begin{array}{l}\text { Microwave or laser radar sensors: Transmission of microwave or laser } \\
\text { signals within the traffic vicinity brings about echoes which help detect } \\
\text { traffic density. }\end{array}$ \\
\hline & & $\begin{array}{l}\text { Infrared sensors: Vehicles are detected by the emission and reflection of } \\
\text { light energy. }\end{array}$ \\
\hline & & $\begin{array}{l}\text { Cameras: Images are taken by the cameras and are used to process the } \\
\text { traffic density. }\end{array}$ \\
\hline
\end{tabular}

The major advantage of using sensors is their close behavior to their real-life counterparts. The sensors are also able to have a record of the past traffic pattern and various occurrences. Therefore, it is possible to model the sensor behavior using these features. The historical data can be used to analyze the performance of the system in order to make decisions on future innovations. The Traffic Control Interface (TraCI) tool, included in the SUMO package enables the WLAN transmission of traffic signals among the various agents. The main steps followed during system execution are:

System START UP: Agent A software application reads the data from the scenario by initializing the on-road embedded sensor

PROCESSING and OUTPUT: It interprets the traffic density signal and determines the pattern of flow (congestion or free flow)

In a congested mode, with the help of the graph indicators, helps agent A know, the location of agent B. As a result, transfers the data acquired in form of a request through the Wireless LAN network.

RECEIVE and PROCESSING: Agent $B$ receives the request, interprets it, process and makes a decision based on the processed signal of the same data.

The decision made is solely to eradicate congestion of agent $\mathrm{A}$ by reducing waiting for time and queue line of motorists in agent $\mathrm{B}$ intersection

RESPONSE and FINAL PROCESSING: The decision encoded is sent back to the requesting agent $\mathrm{A}$ as a response to its request. This response is used to ascertain the current flow.

Agent A checks for the traffic density and then if low terminates the simulation process, else, the simulation process repeats itself. Fig. 2 below illustrate the system execution the study design.

\section{B.System Evaluation}

The ATC is evaluated by using qualitative research method where six respondents were interviewed. The Six respondents include two officials from the traffic division of the Police Force, Ogun State TRACE personnel and road users. The interview sessions were done on the field which went on for at least 20 minutes where various points were taken on the ATC system design. Summary of the evaluation sessions were presented below in the following sub-sections

\section{Interview Question 1 (Positive expectations for the use of ATC)}

It was agreed that ATC will give positive expectations as expressed by one of the Police Traffic official that:

"When this ATC is installed, it relieves the pain that we are traffic officials are facing; it is not easy to be using our hands to be controlling. " He further emphasized "Hey! Stop!, Hey! Come!, Hey! Go!. You use your eyes, you use your mouth, you use your leg, all your body you use it to be controlling." He further said: "But when there is a traffic light, it reduces the pain, the stress in every way possible and you feel relaxed, It is good!"

This is also supported by one of the State traffic warden when he said:

"Our positive expectations are to see that traffic lights and other traffic gadgets are put in place to enhance the performance of our job. Take for instance the traffic lights at strategic places in Ogun state, no driver contravene traffic law. But on the contrary, on our paths here, they contravene because we use our physical body and mental ability to control traffic."

\section{Interview Question 2 (ATC and Traffic Control)}

Respondents were asked about the effects of the implementation of ATC on the performance of traffic control. One of the respondents expressed that:

"As I have said earlier, if you see other places in the metropolis of Abeokuta here where the ATC system is being testing shows improvement on the flow of traffic"

This is buttressed by one of the study respondents that: "This ATC system will really help in the area of drivers' compliance to the traffic light regulation.

Technology like this with abilities to keep track of vehicular movement in order

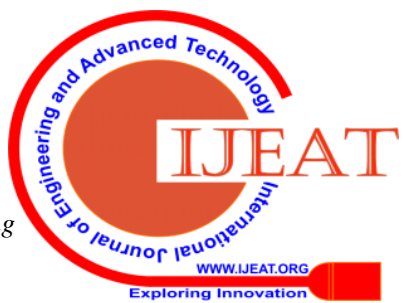


to detect those that fail to comply with the regulation is really a nice idea."

Another view shows that:

"It will be very effective and advantageous if more form of technology can be embedded into ATC system" He further gave a significant example: "ATC with ability to track the locations of traffic congestion in order to inform road users ahead on the best route to their destinations."

\section{Interview Question 3 (ATC communication and Connectivity)}

It was pointed out that the ATC interconnectivity and communication is the most imperative feature of the system. For instance, this was mentioned by one of the respondents that:

"The most vital solution in the ATC is its ability to communicate and interconnect in order to regulate road users ' movement in and out of a particular area."

A similar position was expressed by another respondent that:

"ATC is programmed with a desired waiting time and throughput time intervals which has traffic environmental knowledge from the traffic officials' currently regulation style in order to know what time they will schedule for the different directional waiting time."

Another of the respondents said that:

"And ATC will reduce traffic congest because of its ability to communicate and send traffic regulation signals to each other in order to better regulate the flow of traffic."

\section{Interview Question 4 (ATC limitations)}

Respondents were asked on their perceptions on likely limitations of the system and the following were discovered. A respondent viewed:

"The first and foremost limitation is that the ATC system cannot be able to sense the entire traffic environment such as on-going road construction which can cause an obstruction for road free flow. Hence, there is need to develop a system that can interact effectively with the environment"

He continued:

"There is need to build a system that can interact with various road obstructions such as on-going road construction, road accident, impatient/intolerant road users, market activities, the ugly habit of vehicular parking, the absence of a pedestrian bridge and others. This will really make the system intelligent in nature."

\section{V.CONCLUSION}

This paper shows that urban traffic in both developed and developing countries where road users have been facing traffic jam, bottleneck and high rate of fatal road accident can have a better experience if ATC system is implemented. The ATC presented in this study is implemented based on the four attributes of an agent-based system namely proactive, reactive, collaborative and negotiation. These four attributes ensures that ATC will be able to interact effectively within its immediate traffic environment in order to control and manage traffic."

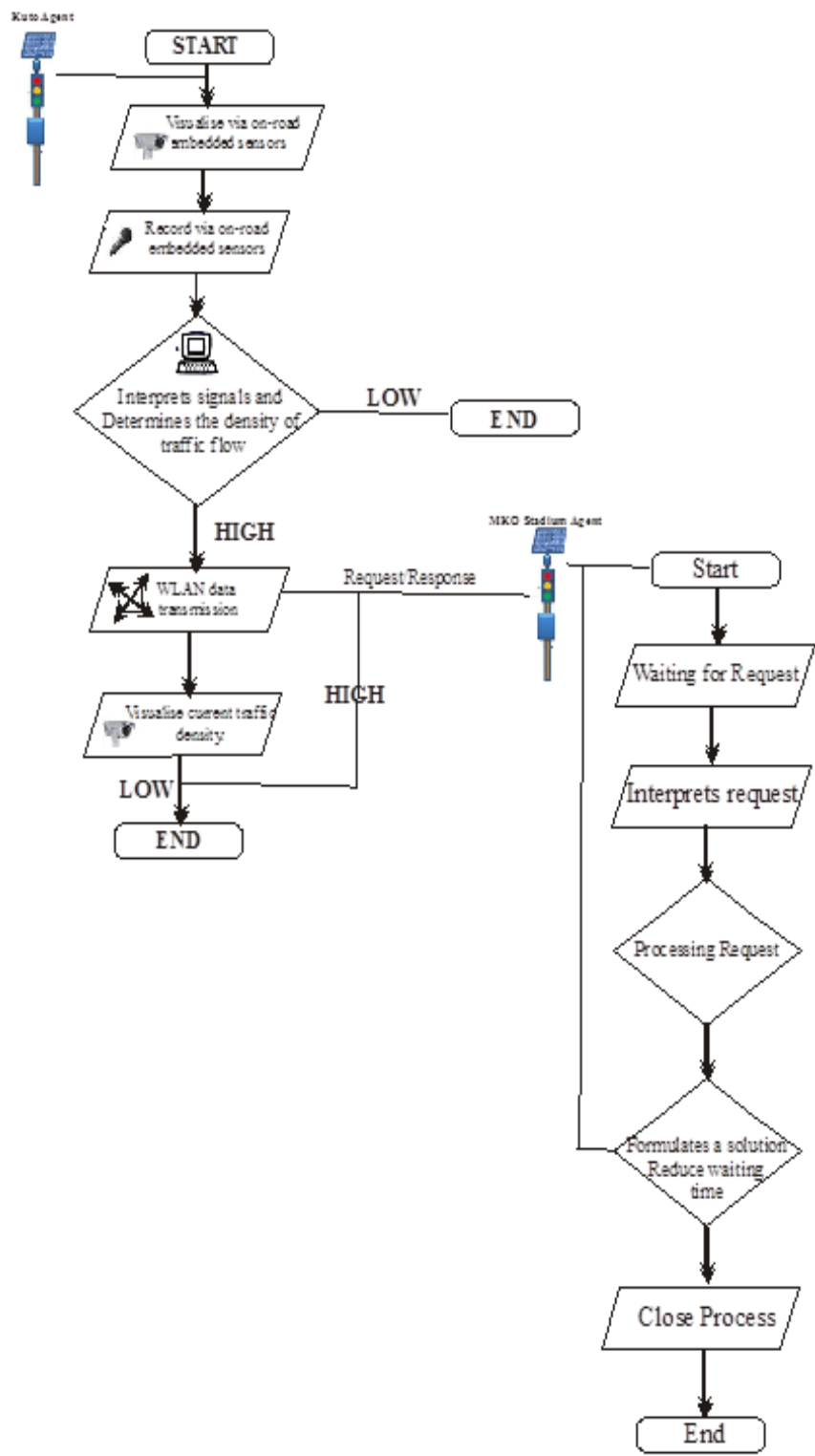

Fig. 2. Agent-based Traffic Control (ATC) System Execution Flowchart

"They (agents with the help of sensors) monitor these intersections by visualizing the environment, recording the various observations, reproduces these observations and performs an action (regulate) by interacting with the other agent based on the same data. Fig. 3 below summarized the system design implementation in this study. 


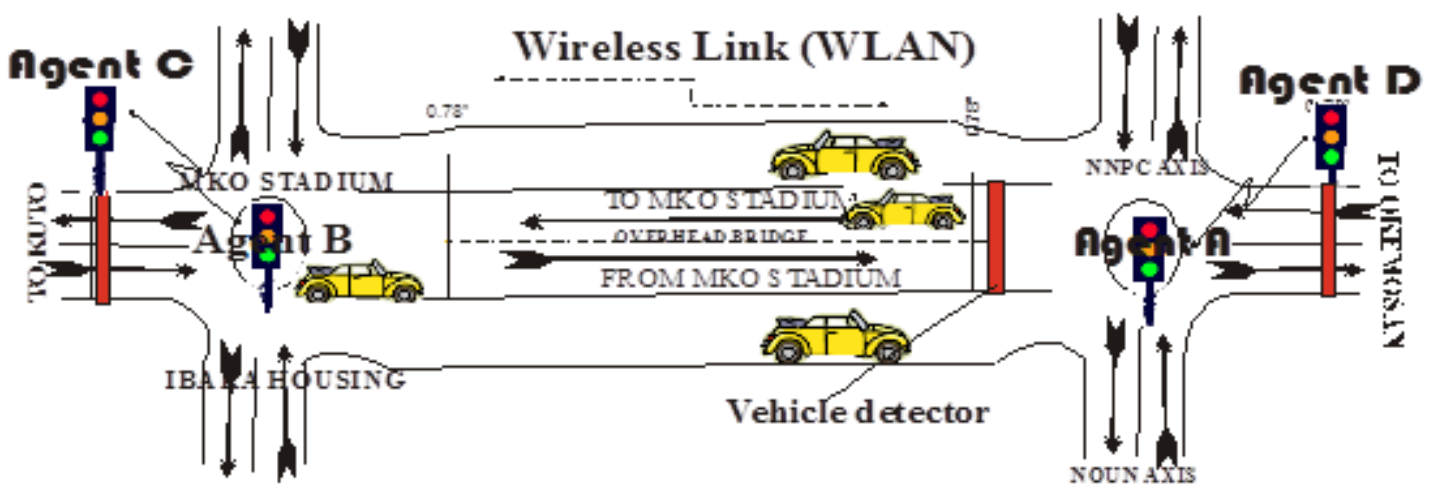

Fig. 3. Agent-based Traffic Control (ATC) System Implementation Layout

\section{REFERENCES}

1. L. Qi, M. Zhou, and W. Luan, “A two-level traffic light control strategy for preventing incident-based urban traffic congestion," IEEE transactions on intelligent transportation systems, vol. 19, no. 1, 2018, pp. 13-24.

2. Y. J. Zhang, A. A. Malikopoulos, and C.G. Cassandras, "Optimal control and coordination of connected and automated vehicles at urban traffic intersections," In 2016 American Control Conference (ACC), 2016, pp. 6227-6232.

3. R. Sundar, S. Hebbar, and V. Golla, "Implementing intelligent traffic control system for congestion control, ambulance clearance, and stolen vehicle detection," IEEE Sensors Journal, vol. 15, no. 2, 2015, pp. 1109-1113.

4. M. Keyvan-Ekbatani, M. Papageorgiou, and V. L. Knoop, "Controller design for gating traffic control in presence of time-delay in urban road networks," Transportation Research Procedia, vol. 7, 2015, pp. 651-668.

5. A. A. Zaidi, B. Kulcsár, and H. Wymeersch, "Back-pressure traffic signal control with fixed and adaptive routing for urban vehicular networks," IEEE Transactions on Intelligent Transportation Systems, vol 17 , no. 8, 2016, pp. 2134-2143.

6. M. Gulić, R. Olivares, and D. Borrajo, "Using automated planning for traffic signals control," PROMET-Traffic\&Transportation, vol. 28, no. 4, 2016, pp. 383-391.

7. P. Maheshwari, P. Kachroo, A. Paz, and R. Khaddar, "Development of control models for the planning of sustainable transportation systems," Transportation Research Part C: Emerging Technologies, vol. 55, 2015, pp. 474-485.

8. C. Ledoux, "An urban traffic flow model integrating neural networks," Transportation Research Part C: Emerging Technologies, vol. 5, no. 5, 1997, pp. 287-300.

9. A. H. Chow, R. Sha, "Performance analysis of centralized and distributed systems for urban traffic control," Transportation Research Record, vol 2557, no. 1, 2016, pp. 66-76.

10. F. Ahmed, and Y. E. Hawas, "An integrated real-time traffic signal system for transit signal priority, incident detection and congestion management," Transportation Research Part C: Emerging Technologies, vol. 60, 2015, pp. 52-76.

11. R. O. Sinnott, L. Morandini, and S. Wu, "SMASH: A cloud-based architecture for big data processing and visualization of traffic data," IEEE International Conference on Data Science and Data Intensive Systems, 2015, pp. 53-60.

12. Y. Lai, F. Yang, L. Zhang, and Z. Lin, "Distributed public vehicle system based on fog nodes and vehicular sensing," IEEE Access, vol. 6, 2018, pp. 22011-22024.

13. S. A. Meshram, and R. S. Lande, "Traffic surveillance by using image processing," In 2018 International Conference on Research in Intelligent and Computing in Engineering (RICE), 2018, pp. 1-3.

14. N. R. Jennings, and S. Bussmann, "Agent-based control systems," IEEE control systems, vol. 23, no. 3, 2003, pp. 61-74.

15. A. Ojeniyi, A. Aziz, and Y. Yusof, "Designing a BDI agent reactant model of behavioural change intervention," Jurnal Teknologi, vol. 78, no. 2-2, 2016, pp. 83-93.

16. A. Ojeniyi, A theoretical and practical approach to a persuasive agent model for change behaviour in oral care and hygiene, $\mathrm{PhD}$ diss., Universiti Utara Malaysia, 2016.

17. A. Ojeniyi, A. Aziz, and Y. Yusof, "Formal analysis of an agent support model for behaviour change intervention," International Journal on Advanced Science, Engineering and Information Technology, vol. 6, no. 6, 2016, pp. 1074-1080.
18. A. Ojeniyi, A. Aziz, and Y. Yusof, "Verification analysis of an agent based model in behaviour change process," In 2015 International Symposium on Agents, Multi-Agent Systems and Robotics (ISAMSR), 2015, pp. 87-92.

19. M. Mullarkey, and A. R. Hevner, "An elaborated action design research process model," European Journal of Information Systems, vol. 28, no. 1, 2019, pp. 6-20.

\section{Authors Profile}

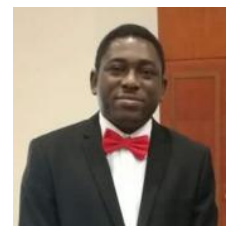

Dr. Adegoke Ojeniyi is a computational scientist with a strong analytical mindset. He has developed various models like Persuasive Agent Model (PAT) and Agent-based Land Slide Disaster Mode. He possess strong background skills in the areas of Mathematical modeling, Artificial Intelligence, Machine learning and Computation analysis Currently, he is the Head of Artificial Intelligence Lab (AiLab) KolaDaisi University. He had his $\mathrm{PhD}$ in Computer Science from Universiti Utara Malaysia

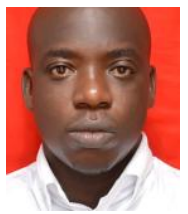

Dr. Taye Aro is a Lecturer with the Department of Computer Science KolaDaisi University. He had his $\mathrm{PhD}$ in Computer Science from Ladoke Akintola University of Technology.

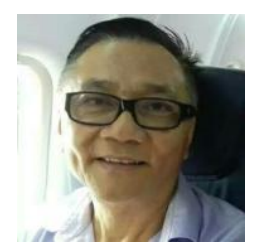

Dr. Ang Moon Thiak is a researcher with Management Information System Research Platform, Othman Yeop Abdullah (OYA) Graduate School of Business, Universiti Utara Malaysia. 